\title{
URBANIZACIÓN MARGINAL Y ASENTAMIENTO OBRERO EN VILLAJOYOSA (1950-1975)
}

\author{
Consuelo Sebastia Llinares
}

\begin{abstract}
RESUMEN
Dentro de la línea actual sobre la promoción inmobiliaria marginal en las ciudades españolas, se pretende, mediante el análisis de casos concretos en Villajoyosa, establecer la caracterización de los agentes y mecanismos que intervienen en la gestación de esta tipología de promociones fragmentarias y descoordinadas, que se convierten en protagonistas del crecimiento urbano. Se plantea la necesidad de una ordenación y la puesta en marcha de medidas que regulen la actuación de los promotores. Posteriormente, el trabajo se centra en la identificación de todo un conjunto de fenómenos implicados en la estructura rústica preexistente, que inciden en las características conceptuales y formales de estos asentamientos.
\end{abstract}

\begin{abstract}
RÉSUMÉ
Dans la ligne actuelle sur la promotion immobilière marginale dans les villes espagnoles on essaie, à Villajoyosa, à travers l'analyse de fans concrets, établir la caractérisation des agents et des mécanismos qui interviennent dans la naissance de cette typologie de promotions fragmentarias et manquant de coordination et qui deviennent les acteurs principaux de la croissance urbaine. Le besoin d'un aménagement et la mise en place de mesures qui régularisent l'action des promotores, se font sentir.

Le travail se centre, ensuite, sur l'identification de tout un ensemble de phénomènes jouant dans la structure rustique préexistante qui influent dans les caractéristiques conceptuelles et formelles de ces lieux.
\end{abstract}

Desde el inicio de los años cincuenta y hasta finales de la década de los setenta, el núcleo urbano de Villajoyosa ha experimentado un gran desarrollo, el mayor de su historia en este corto período de tiempo. Se transmuta el lento crecimiento que caracteriza a las centurias precedentes y primera mitad del siglo actual, por un dinamismo espacial que bien puede calificarse de "explosivo", fiel reflejo de la pujante trayectoria demográfica: el municipio 


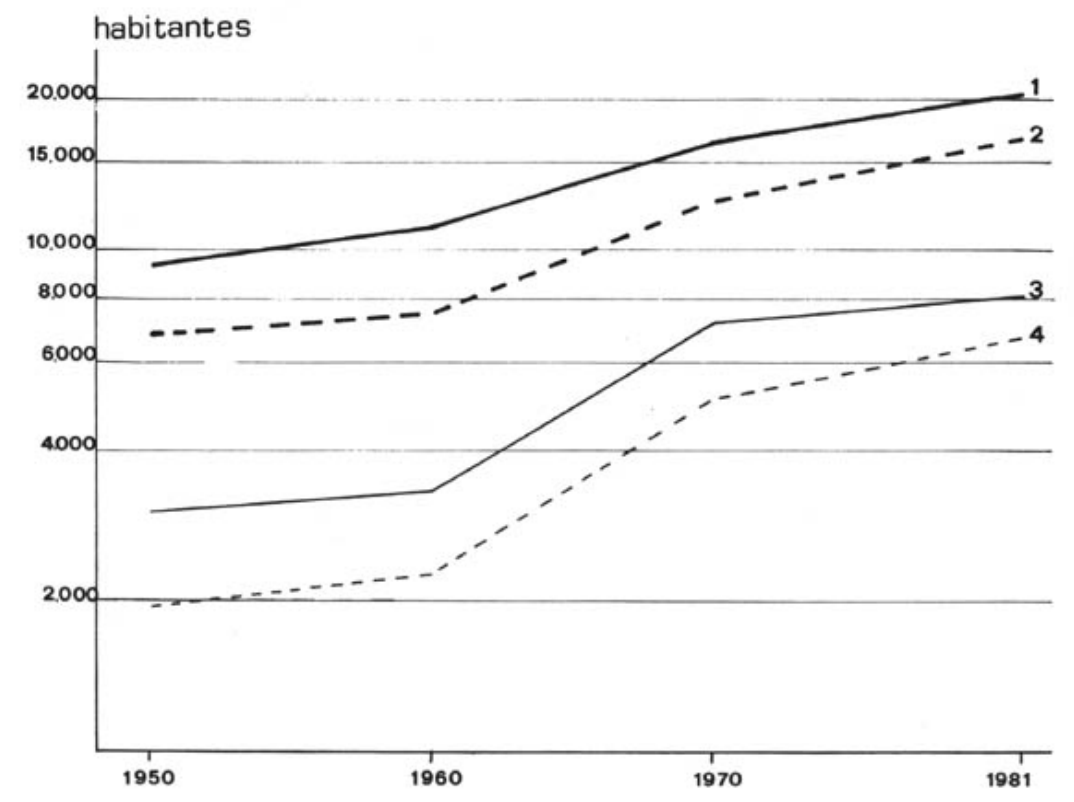

FIGURA 1.: Evolución de la población y del parque inmobiliario de Villajoyosa desde 1950 a 1981: 1, Población total del municipio: 2, Población del casco urbano; 3, Total de viviendas en el municipio; 4, Viviendas en el casco urbano.

pasa de 9.315 habitantes a mediados de la centuria a 20.638 treinta años después, debido, en gran medida, al incremento del casco urbano en más de diez mil moradores, pues pasa de 6.817 a 16.846 en dicho período.

Las profundas transformaciones socio-económicas acaecidas en las últimas décadas, han dado lugar a una variada gama de posibilidades económicas, lo que coadyuva a una diversificación en la edificación de carácter residencial, atendiendo a las necesidades de las distintas clases sociales; el espacio residencial y la calidad de la vivienda han pasado a ser uno de los elementos más diferenciadores entre los estratos sociales, repercutiendo en la ciudad no sólo en la expansión superficial, sino en los diferentes tipos de crecimiento, morfología y calidad urbana.

Así, la ampliación del entramado urbano de la ciudad hay que enmarcarlo en un doble contexto: el continuo urbano estructurado en tomo a la convergencia de los viales básicos (1), que ha quedado consolidado como el área residencial de las clases acomodadas, actuando como el centro estructurador y director del planeamiento urbanístico; por otro lado, la creación de asentamientos marginales al desarrollo urbano convencional -en sus orígenes "vanguardia" del espacio urbanizado y que constituyen en la actualidad un envolvente al casco urbano-, que proliferan ante la existencia de una demanda poco solvente que no puede 
Cuadro I

EVOLUCION DE LA POBLACION Y DEL PARQUE INMOBILIARIO DE VILLAJOYOSA (1950-1981)

\begin{tabular}{lcccccccc}
\hline \multicolumn{1}{c}{$\begin{array}{c}\text { POBLACION DE HECHO } \\
\begin{array}{c}\text { CASCO } \\
\text { URBANO }\end{array}\end{array}$ INDICE } & \%* & $\begin{array}{c}\text { TOTAL } \\
\text { MUNICIPAL }\end{array}$ & $\begin{array}{c}\text { CASCO } \\
\text { URBANO }\end{array}$ & INDICE & \%* & $\begin{array}{c}\text { TOTAL } \\
\text { MUNIC. }\end{array}$ \\
\hline 1950 & 6.817 & 100 & 73,2 & 9.315 & 1.949 & 100 & 64,7 & 3.013 \\
1960 & 7.508 & 110,1 & 68,2 & 11.006 & 2.286 & 117,3 & 69,0 & 3.312 \\
1970 & 12.573 & 184,4 & 77,3 & 16.258 & 5.131 & 263,2 & 71,3 & 7.195 \\
1981 & 16.846 & 247,1 & 81,6 & 20.638 & 6.880 & 353,0 & 84,7 & 8.119 \\
\hline
\end{tabular}

acceder al mercado normal de las viviendas. En el caso de Villajoyosa, los grupos de viviendas "baratas" edificados entre 1953 y 1970 suman un total de 1.425, con una superficie ocupada de $41.032 \mathrm{~m}^{2}$; es decir, este tipo de viviendas supone en Villajoyosa el 8,8 \% del total existente en el municipio en 1970, lo que las eleva a rango harto representativo en esta ciudad litoral. En su mayoría, como indica el Cuadro III, se trata, obviamente, de viviendas de reducida superficie, pues el $28,4 \%$ no alcanza los $60 \mathrm{~m}^{2}$, mientras que este porcentaje se eleva al $77,2 \%$ si consideramos aquellas viviendas que están por debajo de $70 \mathrm{~m}^{2}$.

\section{Identificación de los asentamientos y promotores}

Al analizar la aparición de las urbanizaciones marginales y la identificación de los agentes reales que con su actividad determinaron la gestación de esta tipología de barrios, cabe destacar la información aportada por los Expedientes de Licencias de Obras (2) que, junto con la identificación del promotor, memoria y planos del proyecto, informan someramente sobre las características de la parcelación del suelo a urbanizar.

Sobre la base de la información obtenida, ha sido posible profundizar y retroceder a la estructura rústica de las parcelaciones a través del acceso a la documentación pertinente del Catrasto de Rústica, que se reduce a un croquis del suelo rústico, levantado en torno a 1945 (3). Junto a la cartografía señalada, también existe una relación de fichas-inventarios de las parcelaciones -titular, superficie, usos, valoración, pormenores y posteriores ventas de las fincas-, que son punto de partida valiosísimo para el seguimiento del desarrollo concreto de la parcelación.

A otro nivel, también hay que resaltar la importancia del trabajo de campo como instrumento básico, que completa y en algunos casos se convierte en el apoyo exclusivo de la investigación.

Atendiendo al marco general del crecimiento urbano y, por tanto, de la creación de viviendas, hay que señalar que con independencia de los Planes de Ordenación de las grandes ciudades tras la aprobación de la Ley del Suelo de 1956, la actividad del planeamiento en los núcleos de menor importancia continuó por acuerdos municipales con la Jefatura Nacional de Urbanismo (4); sin una definición explícita del ordenamiento jurídico que asesorara y revisara la actuación de los promotores a una clara orientación unitaria de ordenación (5). De 
ahí, que la insatisfecha demanda de viviendas baratas en la ciudad se materialice en una variada tipología de promociones fragmentarias y descoordinadas.

\section{La carencia de viviendas baratas y las actuaciones de las instituciones locales}

a) El primer grupo de viviendas económicas surge alrededor de 1951 (6) con la creación de un grupo para pescadores denominado popularmente El Pòsit, ubicado en la fachada marítima $\mathrm{y}$ en las inmediaciones del contradique. Se trata de un conjunto de veintiocho viviendas financiadas por la Cofradía de Pescadores local, con el objeto de solventar el acuciante problema de falta de casas para la población menos favorecida del subsector pesquero. Los edificios, alineados en bloques formando "peine", presentan en exceso los defectos de las actuaciones de viviendas baratas de empresa, que busca formas económicas de edificación sin pensaren el futuro ni en el desarrollo del nivel de vida del trabajador, lo que se ha plasmado hoy en graves problemas de calidad de vida (7). En este proyecto privó más la idea caritativa que la de una verdadera promoción social.

El objetivo de este organismo era la administración directa de las viviendas, con poder de decisión sobre las concesiones y mantenimiento. De ahí, que la forma de acceso de la población marinera para la ocupación de aquéllas ha sido desde el principio un contrato privado de inquilinato -de carácter vitalicio- extensible a la viuda del ocupante. La cancelación de dicha concesión queda restringida a dos situaciones concretas: el cambio profesional del arrendatario o la posesión por el mismo de otra vivienda en propiedad. Sin embargo, en los últimos años, se han desarrollado una serie de conflictos entre ambas partes contractuales. La valoración del espacio litoral sobre el que se ubican estas viviendas las han convertido en focos de especial interés para una cierta especulación, a pesar de sus manifiestas deficiencias arquitectónicas.

Así, en ocasiones, los arrendatarios que han elevado considerablemente su nivel económico y han tenido acceso a la propiedad de otras viviendas, conservan sin embargo el alquiler y siguen residiendo en las casas de la Cofradía -que debían tener una eminente función social- por diversos motivos, entre los que destacan la ya comentada valoración de la costa y la costumbre adquirida de vivir en ellas. En estos casos, se ha llegado a la situación de que algunos arrendatarios han alquilado su vivienda en propiedad a los precios especulativos del veraneo tradicional, mientras que ellos siguen pagando una mensualidad simbólica establecida en 200 ptas.(8).

b) Evidentemente la apremiante necesidad de viviendas económicas no fue mitigada por esa modesta actuación, de ahí que, en la segunda mitad de la década de los cincuenta, de nuevo la Cofradía de Pescadores se atribuyera la responsabilidad de impulsar la construcción con carácter urgente de una nueva barriada. En este caso, la solución se encontró en la protección oficial, cuya actuación no fue directa, sino canalizada a través del Instituto de la Marina. Aunque se mantuvo la concepción paternalista, se propugnó, no obstante, el régimen de acceso a la propiedad para estimular de ese modo el incremento del nivel de vida de los trabajadores del mar.

Se construye el barrio del Carmen, localizado en la periferia del casco urbano, sobre suelo rústico -improductivo y en baldío- aledaño a la N-332 en su salida hacia Benidorm. Esta 


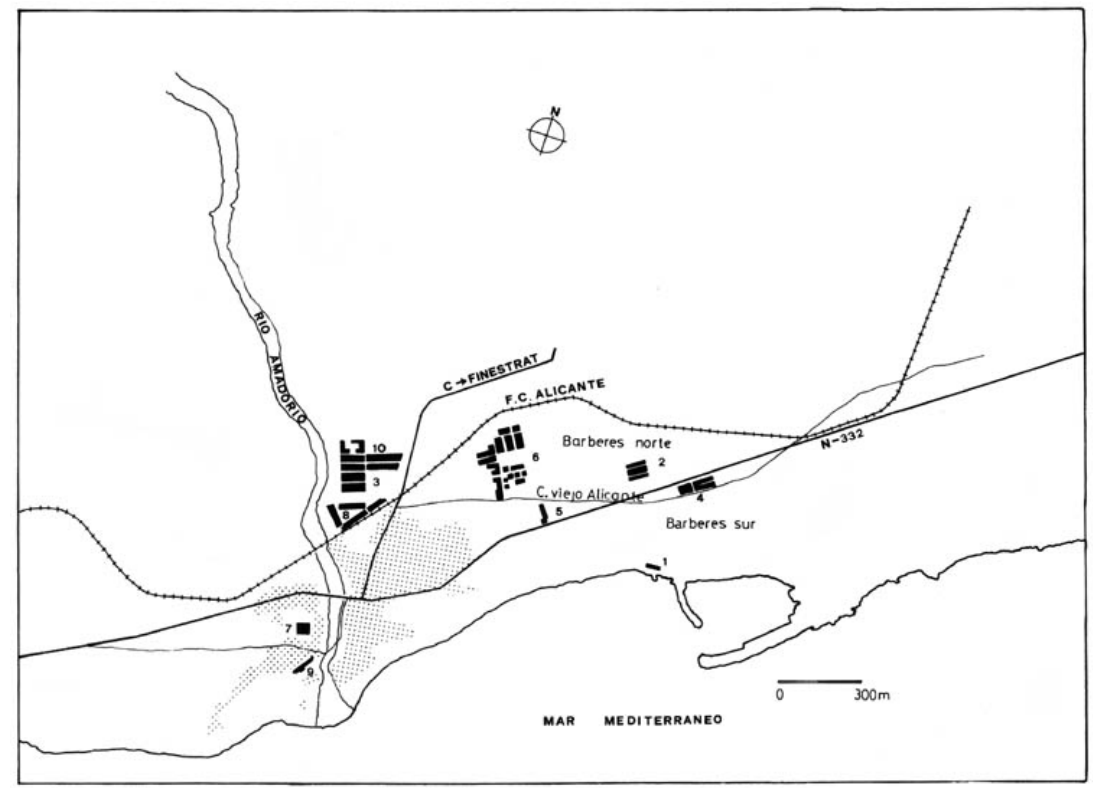

FIGURA 2.: Localización de las áreas de estudio (en punteado el casco urbano consolidado en 1956): 1, Casas del Pòsit; 2, Barrio del Carmen; 3, Barrio de Les Casetes; 4, Bloques Soler; 5, Sureste; 6, Barrio del Pàti Fosc; 7, Nuestra Señora de la Salud: 8, Plaza de la Comunidad; 9, Méndez Núñez: 10, Sector Noroeste. 
Cuadro II

INDIVIDUALIZACION DE LAS PROMOCIONES

\begin{tabular}{|c|c|c|c|c|c|}
\hline $\begin{array}{l}\text { FECHA DE } \\
\text { PROMOCION }\end{array}$ & DENOMINACION & PROMOTOR & $\begin{array}{l}\text { SUPERF. } \\
\text { OCUPADA. }\end{array}$ & $\begin{array}{c}\mathrm{N}^{\mathrm{e}} \\
\text { VIV. }\end{array}$ & $\begin{array}{l}\text { TIPOLOGIA } \\
\text { RESIDENC. }\end{array}$ \\
\hline 1953 & $\mathrm{~B}^{\mathrm{o}}$ Pòsit & Cof. Pesc & 800 & 28 & Bloque Alt. \\
\hline 1957 & $\mathrm{~B}^{\circ}$ Carmen & Cof. Pesc. & 2.850 & 31 & Casa-baja \\
\hline 1959 & $\mathrm{~B}^{\circ}$ Casetes & Ayuntam. & 4.500 & 48 & Casa-baja \\
\hline 1962 & $\mathrm{~B}^{\circ}$ Soler & M. Soler & 4.349 & 173 & Bloque Alt. \\
\hline 1963 & Sureste & Coop. SE & 1.193 & 40 & Bloque Alt. \\
\hline \multirow[t]{3}{*}{1963} & $\mathrm{~B}^{\circ}$ Pàti-Fosc & M. Soler & 6.000 & 284 & Bloque Alt. \\
\hline & & M. Ortiz & 5.850 & 276 & Bloque Alt. \\
\hline & & INVISA & 4.900 & 174 & Bloque Alt. \\
\hline \multirow[t]{2}{*}{1964} & $\mathrm{~B}^{\circ}$ N.S.Salud & Coop.N.S. & & & \\
\hline & & Salud & 1.900 & 60 & Bloque Alt. \\
\hline \multirow[t]{2}{*}{1964} & $\mathrm{~B}^{\circ}$ Plaza & Comunidad & & & \\
\hline & Comunidad & Regantes & 7.600 & 272 & Bloque Alt. \\
\hline \multirow[t]{2}{*}{1970} & $\mathrm{~B}^{\mathrm{o}}$ Mendez- & Construc. & & & \\
\hline & Nuñez & Aragonés & 1.090 & 39 & Bloque Alt. \\
\hline TOTALES & & & 41.032 & 1.425 & \\
\hline
\end{tabular}

* Metros cuadrados

Fuente: Expedientes de Licencias de Obras del Archivo Técnico del Ayto. de Villajoyosa. Elaboración propia.

Cuadro III

CLASIFICACION DE LAS VIVIENDAS SEGUN LA SUPERFICIE HABITABLE

\begin{tabular}{|c|c|c|c|c|c|}
\hline \multicolumn{6}{|c|}{ SUPERFICIE $\left(\mathrm{m}^{2}\right)$} \\
\hline DENOMINACION & $<60$ & $61-70$ & $71-80$ & $81-90$ & $>90$ \\
\hline Pòsit & 28 & - & - & - & 一 \\
\hline Carmen & 31 & 一 & - & - & 一 \\
\hline Casetes & - & 48 & - & - & 一 \\
\hline Soler & 25 & 148 & - & - & 一 \\
\hline Sureste & 10 & 20 & 10 & - & 一 \\
\hline Pàti Fosc $1^{\underline{a}} \mathrm{~F}$. & 112 & 112 & 54 & 6 & 一 \\
\hline Pàti Fosc $2^{\mathrm{a}} \mathrm{F}$. & - & 48 & - & - & 一 \\
\hline Pàti Fosc $3^{\mathrm{a}} \mathrm{F}$. & 24 & 204 & - & - & 一 \\
\hline Pàti Fosc $4^{a} \mathrm{~F}$. & 174 & - & - & - & 一 \\
\hline $\mathrm{N}^{\mathrm{a}}$ Sra. Salud & - & - & - & 一 & 60 \\
\hline Plaza Comunidad & - & 80 & 144 & 48 & 一 \\
\hline Méndez Núñez & 1 & 35 & 3 & - & 一 \\
\hline Total & 405 & 695 & 211 & 54 & 60 \\
\hline
\end{tabular}

Fuente: Expedientes de Licencias de Obras del Archivo Técnico del Ayto. de Villajoyosa. Elaboración propia. 
agrupación de treinta y una viviendas desborda la primera línea de la citada carretera, creando ciudad en paralelo hacia el interior. Por otra parte, esa estrategia de urbanización marginal en "segunda línea", en suelo con menores expectativas de especulación, permitió una edificación horizontal, construyéndose viviendas unifamiliares para evitar el choque frontal con las condiciones rurales del entorno, intentando dar una fisonomía de "ciudad-jardín", aunque nunca lo fueran.

c) Coetáneo al proyecto descrito, la Corporación Municipal de Villajoyosa, que contaba con el apoyo legal de la Ley de Régimen Local, de acuerdo con la normativa del Consejo Provincial de la Vivienda y del Patronato Francisco Franco (9), solicitó del Ministerio de Hacienda la concertación de un préstamo, al mismo tiempo que aprobó su primer presupuesto especial de urbanismo (10) para constituir un Patrimonio Municipal de Suelo, libre de cargas. De ese modo, la euforia constructora del Ayuntamiento se plasmó en un grupo de cuarenta y ocho viviendas protegidas que engloban el barrio denominado Les Casetes, alusión harto significativa a su formación. Este conjunto, dentro del subtipo edificatorio de casas bajas, se individualiza por la pérdida del pequeño jardín delantero, con la consiguiente degradación del pretendido formato de "ciudad-jardín" que, como garantía de modernidad, intentaba acercar el campo a la ciudad. No obstante, esta manifestación presenta una gran relevancia, conferida por el importante cambio de dirección geográfica que se imprime en la ubicación de las viviendas obreras; se da preferencia a la ocupación del espacio periférico más cercano al ensanche convencional. Se edifica sobre terreno marginal entre la carretera comarcal a Finestrat y la línea del ferrocarril de Alicante a Denia. Posteriormente, este núcleo se constituirá en el primer foco urbanizador y eje director del desarrollo urbano de la ciudad.

\section{La labor del estado: el Instituto Nacional de la Vivienda.}

En la década de los sesenta, la actuación del Estado por medio de la iniciativa privada se hace cada vez más importante, debiéndose admitir que la legislación urbanística posterior a 1954 se orientó mayoritariamente hacia la construcción de viviendas destinadas a la clase obrera. Esta estrategia constructora mantenida por el Gobierno, ha hecho de la política de la vivienda argumento político, cayendo en fáciles triunfalismos y en la ausencia de autocrítica (11). Se adolece, en general, de coordinación en el planeamiento, olvidándose a menudo las implicaciones urbanísticas y sociales para su completa inserción en un crecimiento urbano armónico.

De la "generosidad" de la protección oficial, será la iniciativa privada quien obtenga la mayoría de los fondos públicos, beneficiándose los promotores particulares de la imperiosa necesidad de viviendas sociales y asequibles; a ello contribuye la aspiración generalizada en las categorías sociales más bajas de poseer una vivienda en propiedad.

Al igual que las actuaciones que se acaban de analizar, estas promociones se localizan preferentemente en la corona periférica, que facilita el mayor tamaño de la parcela que se pretende urbanizar, a la vez que evita el encarecimiento de la vivienda por los abusivos precios del suelo calificado de uso urbano. Los diferentes promotores, cada uno de acuerdo con unos intereses concretos, promovieron una edificación maciza que aprovecha al máximo el espacio, con conjuntos de bloques rectangulares que se disponen alineados o en forma paralela, lo que contribuye a aumentar la monotonía del conjunto, facilitando su identificación dentro del entramado urbano. 
a) La primera actuación se asienta entre la N-332 y el antiguo camino de Alicante -sector de Barberes Sur-, con la construcción de dos macizas moles de parecida fisonomía, que rompen la visión unitaria del tradicional hábitat rural de casas bajas inmediatas a la carretera y destacan sobre los extensos espacios aún libres del entorno.

Este barrio, que totaliza ciento setenta y tres viviendas subvencionadas, fue promovido por la constructora Manuel Soler Soriano en el primer trienio de los sesenta. Ese proyecto será el primer modelo de la labor constructora del citado promotor, que constituirá el caso más relevante y prototípico de mente inmobiliario foráneo con mayor capacidad de actuación(12).

b) En ese mismo período se aprueba la construcción de una nueva barriada obrera en la ciudad, El Pàti Fosc, que busca un emplazamiento más cercano a la zona urbana consolidada, ocupando terrenos periféricos vacíos y constreñidos entre el viejo camino a Alicante y el trazado de la línea férrea al norte. No obstante, hay que indicar que la inmejorable localización en el límite del área del ensanche no fue suficiente para impulsar una racional planificación del barrio. El trazado interno del conjunto queda reducido a la simple superposición en el tiempo y en el espacio de actuaciones parciales de diferentes promotores, rompiendo la imagen ortogonal del ensanche.

c) De manera simultánea a la gestación de esas promociones y al amparo del grupo de viviendas denominado Les Casetes, se inicia la expansión urbana por el sector noroeste del núcleo, una vez superado el obstáculo que supone el ferrocarril. Surge así el barrio designado como "La Plaza de la Comunidad" o "la Sicop", acogiéndose a la denominación de la empresa constructora: se levantan cuatro bloques que aglutinan doscientas setenta y nueve viviendas. La disposición de los inmuebles se adapta a la forma triangular de la parcela, que genera un espacio vacío interior reservado como centro de esparcimiento. La relevancia de esta actuación radica en una pluralidad de factores, como son: su ubicación en un solar de conexión con el ensanche, aspecto que ha repercutido en un mayor grado de integración de esos asentamientos en el área urbana consolidada: la mejora en la calidad de la vivienda e infraestructuras, que las hace más atractivas y aumenta su prestigio social, pretendiéndose evitar la homogeneidad social que caracteriza Ia promoción oficial, y la unidad de esa comunidad, con la disociación de las viviendas respecto a las vías perimétricas que las circundan: buena prueba de ello es que de un total de dieciocho portales con que cuenta la agrupación, diecisiete se abran a la plaza central, evitando el espacio libre exterior.

Cabe incluir también, dentro de la esfera oficial del negocio inmobiliario, a las cooperativas inmobiliarias, cuya intervención destaca más por el carácter benéfico-social de esas instituciones, que por el volumen de construcción de las mismas, dejando en un segundo plano el ánimo lucrativo que privaba en los proyectos descritos.

Esas entidades urbanizadoras dan preferencia a la promoción de áreas residenciales en los calveros del suelo urbano, vacíos del recinto urbano que acogen infraestructuras y servicios propios de la ciudad. El único acondicionamiento para su uso urbano se reduce a la apertura de zonas de paso que se ajusten a Iza red viaria colindante.

d) En esta línea cabe destacar la actuación de la Constructora Benéfica del Sureste de España que, con capital financiero de la Caja de Ahorros homónima y bajo el protectorado del Gobierno, promovió la construcción de un bloque lineal de cuarenta viviendas de renta limitada, inmediato a la N-332. Esa edificación supuso el reajuste del trazado vidrio programado, con un desplazamiento de la calle en proyecto -que cruzaba el terreno a edificar- 
hacia su periferia, respetándose con esa propuesta las alineaciones de las fachadas preexistentes. La finalidad de la reforma era el mejor aprovechamiento del solar para su uso edificatorio, contando con el apoyo unánime de la Comisión Técnica del Ayuntamiento.

e) Dentro de esa modalidad, algo mayor fue la aportación de la Cooperativa Nuestra Señora de la Salud, que construyó sesenta viviendas en el espacio intermedio que había permanecido sin edificar entre las calles Quintana -paralela al eje de la N-332 a su salida hacia Alicante- y el barrio del Poble Nou, arrabal decimonónico que conserva su ancestral fisonomía morfológica y social. Aunque la tipología edificatoria es la propia de las categorías socio-económicas bajas -bloque en altura que configura una manzana cerrada-, sin embargo, la superficie media de las viviendas y la mejor calidad en los materiales denotan su orientación hacia un mayor equilibrio social.

Como cuestión final de este apartado dedicado a la promoción oficial, hay, que indicar que su actuación en la ciudad no se reduce a las agrupaciones de viviendas analizadas, sino que ha sido mucho más pródiga y con una variada gama de matices arquitectónicos. No obstante, estas variantes no logran encubrir la deficiente aparencia externa y las pésimas características urbanísticas que siguen manteniendo los bloques de viviendas ocupados esencialmente por las clases populares con rentas más bajas.

f) Una muestra evidente de esa actuación es el edificio levantado en el inicio de la década de los setenta en la calle de Méndez Núñez -antiguo camino vecinal que cerraba por el sur el barrio del Poble Nou-. Se trata de un sobrio inmueble de cuatro plantas con la típica forma acuartelada, paredón de cemento que desmantela la fisonomía del barrio y acentúa irremediablemente el acusado proceso de degradación urbana y pérdida de centralidad que caracteriza al Poble Nou desde el trazado exterior de la N-332. Por sí sola, esa edificación premeditada en primera línea del mar, es paradigma de la carencia de un adecuado planeamiento urbano en Villajoyosa.

\section{La estructura de la propiedad rústica y su influencia sobre el urbanismo}

No se pretende con este epígrafe hacer un balance completo del proceso de ocupación de las tierras rústicas de la periferia, sino destacar, de ese conjunto, las ideas caracterizadoras y algunos hechos concretos que sirven de marco de referencia para encontrar las filiaciones y paralelismos de los asentamientos marginales.

Del análisis de las solicitudes de urbanizaciones que han prosperado fuera del perímetro urbano, se desprende que la fuerza de decisión en la elección de los terrenos la ha dado el módico precio del suelo rural. Ese incentivo -dentro de la lógica de la maximización de los beneficiosha afectado por igual a la actuación de los organismos oficiales como a la de los particulares, congelando el desarrollo preferente del ensanche consolidado y forzando el periférico, dejando vacíos los sectores próximos y de contacto, que adquieren así una futura revalorización.

El proceso de asentamientos marginales nacía con la ocupación de pequeñas fincas rústicas disformes y con escaso valor agrícola aunque sean de regadío, susceptibles por tanto de ser convertidas en suelo urbano. Esos terrenos, articulados en torno a los ejes de comunicación, responden a las típicas formas longueras, adaptándose la trama urbana actual a la estructura del parcelario rústico preexistente. 
El principio general que rige es el del aprovechamiento máximo del espacio edificable, con la ausencia de espacios abiertos, salvo los reservados a viales entre las manzanas. De ahí, el ajuste de la alineación de fachadas al borde mismo del solar ocupado, manteniéndose los caminos linderos y zonas de acceso al mismo para su inminente transformación en calles. Se produce de ese modo una jerarquización en el tamaño de las mismas, que con frecuencia incumplen las reglamentaciones de las normativas municipales vigentes, contando sin embargo con la permisibilidad de la Administración local. Los caminos perimétricos al barrio se convierten en las calles principales, con un mayor tamaño y anchura que las secundarias; estas últimas se abren bien mediante un plano ortogonal si la superficie lo permite, o bien mediante el trazado de una simple calle central en el caso de longueros mínimos. En las calles de primer orden las edificaciones suelen distar diez metros entre sí, reduciéndose a ocho metros en el segundo caso.

Ahora bien, estas actuaciones exigen algunos matices en cuanto a las trazas y morfología urbana resultante, como pone de manifiesto el análisis de dos subtipos representativos:

a) La promoción de los bloques "Soler", constreñidos entre el eje de la N-332 y el camino viejo a Alicante, constituyen el ejemplo más perfecto de una sola actuación sobre una estructura de la propiedad rústica distribuida en reducidas parcelas con formatos irregulares. Los propietarios del solar afectado, previo acuerdo con el promotor, permutaron la propiedad jurídica del terreno por el $20 \%$ de las viviendas proyectadas. Se levantan dos bloques cerrados que conforman sendas manzanas, separadas por una calle central de segundo orden, que presenta los problemas de asfaltado propios de este tipo de asentamientos. En el caso concreto que nos ocupa, no es extraño que en situaciones excepcionales de avenida el firme asfáltico presente grietas y socavones propios de los aludidos rasgos de construcción, a los que se aúnan los problemas derivados de su localización en una zona de avenamiento que concentra la escorrentía habitualmente difusa de las laderas.

La respuesta de la autoridad competente se resuelve renovando el asfaltado de los viales en unas condiciones similares, sin atenuarse los problemas de desagüe, que de nuevo serán incapaces de resistir un episodio de avenida (13).

b) Por el contrario, el barrio del Pàti Fosc supone la ocupación de pequeñas fincas rústicas por diferentes promotores, careciendo de un proyecto de regulación que orientara y determinase un desarrollo racional del mismo; se evidencia la marginalidad de su concepción y la dejadez de las normas urbanísticas.

El suelo rústico era considerado como un espacio "amorfo e independiente" capaz de soportar cualquier actuación "suelta" y con un escaso tratamiento urbanístico-ambiental. Por todo ello, la configuración y trazado del barrio queda vinculado de manera directa a las pretensiones de cada empresa promotora, con actuaciones segregadas que no logran borrar las huellas del primitivo parcelario rústico.

En la primera fase de ocupación del espacio se adoptó la solución tradicional de la calle lateral -acomodándose al camino rural existente- como arteria vertebradora del desarrollo longitudinal N-S de la promoción. Si bien, la correspondencia del tamaño de la parcela con la superficie edificable -atendiendo a la aludida base económica- determinaba la desorganización de la trama interna del conjunto. En un primer tramo del eje director se levantó una edificación en línea, abriéndose calles simétricas y transversales al mismo al ampliarse la superficie afectada; estas últimas se cortan al llegar al límite físico de la propiedad. 


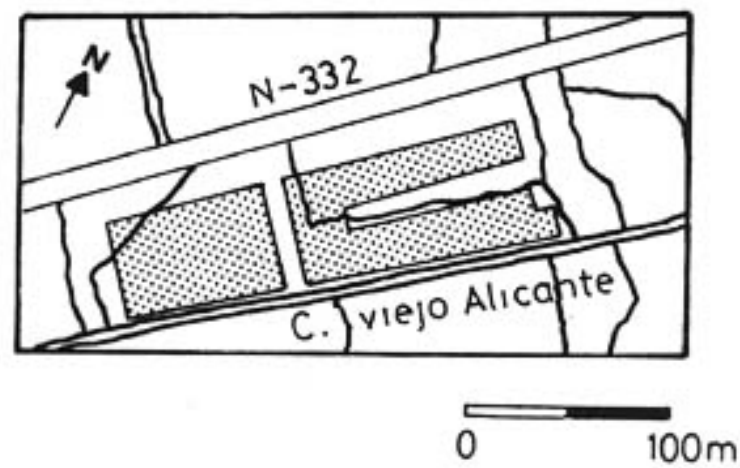

FIGURA 3.: Estructura de la propiedad rústica hacia 1945 en el barrio Soler. Fuente: Catastro de Rústica.

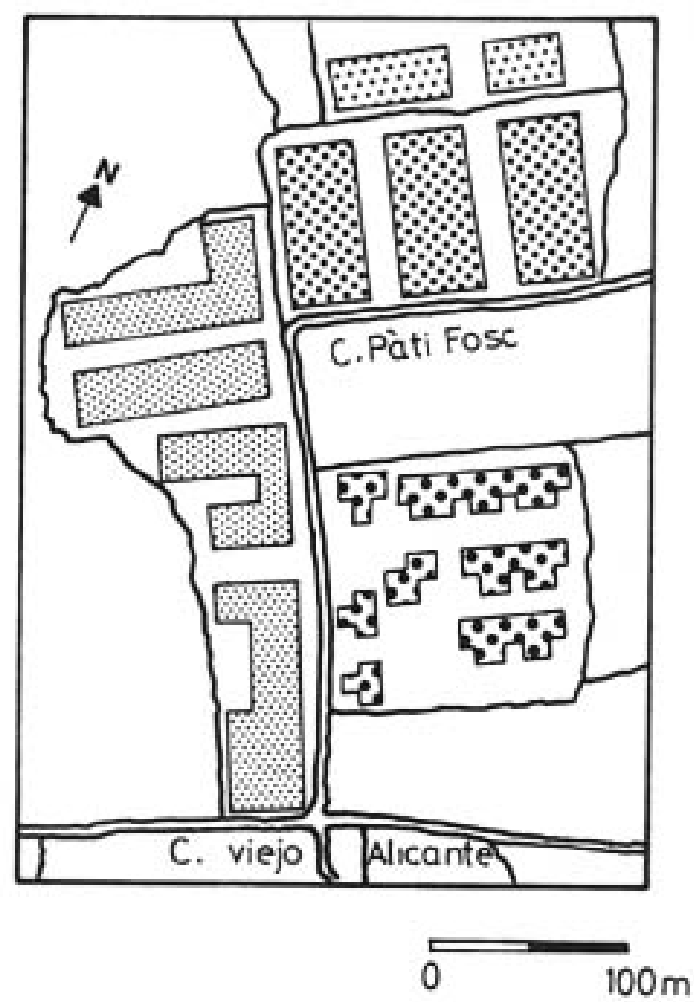

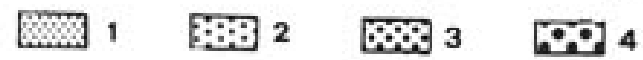

FIGURA 4.: Estructura de la propiedad rústica hacia 1945 en el barrio del Pàti Fosc: 1, $1^{\mathrm{a}}$ Fase; 2, $2^{\mathrm{a}}$ Fase; 3, $3^{\mathrm{a}}$ Fase; 4, 4ª Fase. Fuente: Catastro de Rústica. 
La segunda actuación se reduce a dos pequeños bloques, paralelos al tendido del ferrocarril, que acotan la urbanización por el norte. Se gesta, de ese modo, una calle con dirección W-E que confiere las futuras pautas de expansión del barrio. No cabe duda que la ubicación de "La Barbera" y los terrenos que la circundan, residencia de la familia Aragonés -grandes hacendados que en esos momentos experimentan el desmantelamiento de antiguos patrimonios-, obstaculiza el lógico desarrollo en sentido oeste, buscando la conexión con el principal eje de ensanche de la ciudad -la carretera hacia Finestrat-.

La tercera etapa expansiva del barrio se materializa en tres manzanas cerradas, uniformes y paralelas, que guardan un desorden estético con el trazado viario ya consolidado.

Por último, como broche revelador de esa anárquica distribución, se combinan los subtipos constructivos de bloques abiertos independientes con inmuebles que eliminan la manzana cerrada por una composición volumétrica libre, que pretenden dar un aspecto novedoso y moderno. Se rompe la uniformidad de las edificaciones macizas, utilizándose el espacio con cierta generosidad, sin encubrir la torpeza y falta de intención en el manejo de ese formato de composición, que pone de manifiesto el ejercicio de aprendizaje que se estaba realizando (14).

\section{Usos del suelo: escasez de equipamientos}

La urbanización marginal se define como una forma de crecimiento del uso residencial permanente (15); este hecho queda perfectamente comprobado en los barrios analizados, donde la escasa relevancia numérica de esas construcciones obliga a que se destinen en su mayor parte como viviendas, reduciéndose la dotación de locales comerciales a unos niveles mínimos. Sin embargo, hay que destacar que esa escasez en los usos no residenciales viene determinada por una síntesis de factores, cuya diversificación genera un proceso de desarrollo diferencial en los barrios analizados.

Así, un primer grupo sin ningún tipo de servicios englobaría las promociones del Pòsit, Carmen, Casetes, Sureste, Nuestra Señora de la Salud y Méndez Núñez. Por otro lado, un segundo grupo lo integrarían las áreas donde existe al menos un comercio que cubre las necesidades básicas de la población residente, en esta situación están los bloques "Soler", Pàti Fosc y la "Plaza de la Comunidad". Por último, se analizará la zona noroeste consolidada a un ritmo acelerado a partir de 1975, cuyo nivel de servicios de consumo puede considerarse satisfactorio, contraponiéndose a los asentamientos ya aludidos.

Es evidente que la antigüedad, la naturaleza formal y la consolidación de estas urbanizaciones condicionan el nivel de usos no directamente residenciales. De este modo, las primeras formaciones marginales -donde predomina Ia vivienda unifamiliar de planta única que genera un gradiente de densidad muy bajo y, por consiguiente, con un ínfimo nivel de demanda-, se caracterizan por la carencia de servicios básicos, lo que obliga a un desplazamiento continuo de sus residentes para satisfacer cualquier tipo de necesidad. Dentro de ese grupo se incluyen también los edificios promovidos por cooperativas que, pese al aumento en el número de plantas, arrojan unas cotas de densidad bastantes bajas, ya que en estos casos suelen convivir las casas

antiguas de una o dos plantas con los bloques modernos. Por otra parte, la finalidad benéfico-social de esas promociones determinó la preferente ocupación de los bajos por viviendas. 
Cuadro IV

DISTRIBUCION DE LOCALES COMERCIALES SEGUN SU USO

\begin{tabular}{|c|c|c|c|c|c|c|c|c|}
\hline $\begin{array}{l}\text { USOS NO } \\
\text { RESIDENCIALES }\end{array}$ & B ${ }^{\circ}$ SOLER & $\%$ & PATI FOSC & C $\%$ & PLZA. COM. & 1. $\%$ & $B^{2} \mathbf{N W}$ & $\%$ \\
\hline \multicolumn{9}{|l|}{ —EQUIPAMIENTOS } \\
\hline bares y restaurantes & 5 & 27,8 & 6 & 24,0 & 2 & 12,5 & 3 & 9,4 \\
\hline tiendas alimentación & 3 & 16,7 & 10 & 40,0 & 7 & 43,7 & 8 & 25,0 \\
\hline comercio especializa. & 2 & 11,1 & 5 & 20,0 & 4 & 25,0 & 11 & 34,4 \\
\hline servicios colectivos & - & 0,0 & 1 & 4,0 & 1 & 6,3 & 4 & 12,5 \\
\hline \multicolumn{9}{|l|}{ —USO INDUSTRIAL } \\
\hline talleres & 6 & 33,3 & 1 & 4,0 & 2 & 12,5 & 6 & 18,7 \\
\hline almacenes & 2 & 11,1 & 2 & 8,0 & - & 0,0 & - & 0,0 \\
\hline TOTAL & 18 & 100,0 & 25 & 100,0 & 16 & 100,0 & 32 & 100,0 \\
\hline
\end{tabular}

Fuente: Trabajo de campo

En segundo lugar, aparece el tipo caracterizado por tener los establecimientos comerciales mínimos de consumo -donde se incluyen las tiendas de alimentación, bares y una reducida gama de comercio especializado-, que comparten la ocupación de la superficie comercial con pequeños talleres y almacenes. Esta diversificación en los usos del suelo se identifica con una expansión más temprana de "compactos conjuntos" edificatorios, que adoptan el bloque de media altura -hasta cinco plantas-, generando una mayor densidad de población. La instalación de estos servicios, en su mayoría por los propios residentes del barrio, se realiza cuando ya consolidada el área, existe una demanda que los pueda cubrir.

La distribución de usos es lógica y similar: el mayor número de locales comerciales se localizan en las principales vías de acceso, buscando complementar la propia demanda interna del conjunto con la del resto del espacio circundante. Así, tanto en los bloques Soler como en el Pàti Fosc, las superficies comerciales se reducen a las fachadas que flanquean la N-332 y el antiguo camino homónimo. Por el contrario, el plan central concebido para el grupo de "La Sicop" determinó la apertura de locales solamente en las fachadas perimétricas a la plaza inscrita, que se pretendía vitalizar como centro cívico de la urbanización.

Atendiendo a la clasificación de las funciones, el grupo "Soler" presenta una distribución más equilibrada en los usos; en este caso su asentamiento más periférico, el carácter temprano de su gestación que ha sufrido posteriormente un estancamiento, la baja calidad residencial y la existencia de naves industriales en el contorno, ha repercutido en la multiplicidad de usos. Los comercios suponen el 55,6 \% lo del total, siendo los bares -uso con mayor rendimiento- los establecimientos preponderantes.

Las actividades de almacenaje y talleres, normalmente segregados de las áreas de calidad, comparten en este sector su marginalidad con una residencia degradada; en la medida en que se acentúa el deterioro de los inmuebles prolifera el uso industrial. Sin embargo, la gran mayoría de locales están infrautilizados y cumplen una función secundaria (almacenaje de muebles, toldos, etc). 
Por el contrario, las agrupaciones del Pàti Fosc y “La Sicop”, localizadas en el borde del ensanche y con un mayor grado de integración en el núcleo, presentan un ajuste similar en la distribución relativa de usos, donde la característica fundamental es la gran importancia que adquiere la dotación de equipamientos y servicios básicos -que superan el $85 \%$-reduciéndose la utilización industrial a unos niveles mínimos.

Por otro lado, el sector de más reciente urbanización, el noroeste, ha visto incrementada notablemente su densidad demográfica con la ocupación total de su espacio edificable. Junto a ello, la dotación comercial y de servicios se ha visto mejorada gracias a la localización de bajos comerciales, a la mejora general del nivel de vida de la zona y al incremento de la capacidad económica de los residentes. En este sentido, destaca la calle Relleu, vía comercial por excelencia, que localiza la mayor capacidad de establecimientos especializados (mercerías, droguerías, zapaterías, papelerías, etc.). Sin embargo, los pequeños talleres, generalmente de reparación mecánica, se instalan en el sector de más reciente urbanización, al norte, buscando el contacto con la zona industrial colindante.

\section{NOTAS BIBLIOGRÁFICAS}

1 La malla urbana constreñida entre la avenida del País Valencià -tramo urbano de la N-332- y las calles Canalejas y Primo de Rivera, -carretera comarcal de conexión entre Villajoyosa y los municipios del interior-, ha quedado configurada desde el inicio de la centuria como la zona de ensanche tradicional de la ciudad, con edificación de alta calidad, manifiesta sobre todo en las fachadas de las grandes arterias reseñadas, decreciendo aquélla con el distanciamiento lineal a estos ejes.

En el Archivo Municipal de la Oficina Técnica de Obras de Villajoyosa se conserva esta fuente desde los años cincuenta, sin embargo, hay que hacer constar que la serie tan sólo aparece completa y catalogada desde 1960 a 1985.

3 Sobre la utilización de esta fuente, hay que señalar que no guarda una correspondencia estricta con la realidad, condicionada ésta por una serie factores, entre los que cabe destacar las numerosas ocultaciones dado su carácter impositivo, así como la carencia de escrituras de compra-venta que certifiquen la legalidad de la propiedad del suelo. No obstante, hasta 1988 la contribución de rústica en Villajoyosa se ha ajustado a este bosquejo.

4 TERAN, F. de., Planeamiento Urbano en la España Contemporánea. Historia de un proceso imposible, Barcelona, 1978, Ed. Gustavo Gili, p. 361.

5 El primer Reglamento General para Régimen del Suelo y Ordenación Urbana en Villajoyosa no fue aprobado hasta el 12 de Julio de 1962, careciendo de unas adecuadas pautas jurídicas que señalen el alcance, contenido y documentos constitutivos de la Normativa Urbanística que prescribe, por lo que en casos de imprevisión, omisión, aplicación e interpretación dudosa, se acoge a la vigente Ley de Régimen de Suelo.

6 Información obtenida en entrevista con el Secretario de la Cofradía de Pescadores de Villajoyosa.

7 Ver en esta línea ROIZ, M., “Zona minera de León”, Ciudad y Territorio, nº 2, (1973), p. 55.

8 De ahí que la Cofradía de Pescadores haya recurrido a mecanismos judiciales para la cancelación de ocho contratos, de los cuales el fallo de cinco ha sido a favor del Pósito, estando el resto en espera de resolución.

9 (A)RCHIVO (M)UNICIPAL (V)ILLAJOYOSA: Libro de Actas de 1955. Sesión en 6 de mayo de 1955.

10 A. M. V., Libro de Actas de 1955. Sesión en 21 de octubre de 1955.

11 VALENZUELA RUBIO, M., "Iniciativa oficial y crecimiento urbano en Madrid (1939-1973)", Estudios Geográficos, nº 173, (1974), p. 618.

La empresa de construcción Soler Soriano y Cía, posteriormente se fusionará con constructores de 
la localidad, constituyéndose la Sociedad Inmobiliaria Villajoyosa S.A. (INVISA), que alternando la promoción oficial con la libre, ha sido la entidad de mayor participación en la ciudad.

13 Ver en esta línea VERA REBOLLO, F. y MARCO MOLINA, J.A., "Impacto de los usos del suelo y erosión en cuencas vertientes del sur del País Valenciano", Investigaciones Geográficas, nº 6, Instituto Universitario de Geografía, Univ. de Alicante, pp. 7-31.

14 TERAN, F. de., ob. cit., p. 294.

15 CANOSA ZAMORA, E. Y RODRÍGUEZ CHUMILLAS, J., "Urbanización marginal en la periferia noreste de Madrid". Ciudad y Territorio, no 4, (1985), p. 34. 